\title{
Tom Thieme \\ Vorreiter wider Willen? \\ Die SED-PDS-Elite und der Systemwechsel in der DDR
}

\section{Einleitung}

Der gemeinhin als »friedliche Revolution « bezeichnete jüngste Systemwechsel ${ }^{1}$ in der deutschen Geschichte liegt mittlerweile knapp ein Vierteljahrhundert zurück, doch ein Konsens über die Bewertung des Endes der SED-Diktatur, über die Rolle der Staatspartei, der Opposition und der demonstrierenden Bevölkerung hat sich nicht überall gebildet. ${ }^{2}$ Das gilt vor allem für Teile der alten Nomenklatur, die sich heute in der SEDPDS-Nachfolgepartei Die Linke organisieren. So erklärte der einstige »Hoffnungsträger « und Ehrenvorsitzende der PDS Hans Modrow 2010: »Wenn wir Demokratie nicht auf die Chance reduzieren, alle vier Jahre zwischen konkurrierenden Parteien und Personen zu wählen, sondern deren repräsentative Funktion betonen, dann war die >Regierung der nationalen Verantwortung die in ihrem Profil politisch breiteste, d. h. repräsentativste demokratische Regierung, die es in der bisherigen deutschen Geschichte gab. ${ }^{3}$ Staunen lässt sich nicht nur über die doppelte Fehlperzeption - diese Regierung war bekanntlich weder eine demokratisch gewählte, noch repräsentierte sie entfernt die DDR-Gesellschaft. Ebenso bemerkenswert ist, dass es nach dem Ende der kommunistischen Regime offensichtlich weder zur Einigkeit bei der Einschätzung der Systemwechsel an sich kam, noch über die Bewertung der verschiedenen Akteure. Das gilt speziell für die Rolle der sozialistischen Parteielite.

Der »Sonderfall« der DDR - wegen der maßgeblich durch den Wiedervereinigungsprozess geprägten Entwicklung - ist im ostmitteleuropäischen Vergleich kein Einzelfall.

1 »Systemwechsel « bezeichnet in seiner politikwissenschaftlichen Bedeutung den Wechsel zu einem anderen Systemtypus. Das umfasst sowohl den Wechsel von einer Demokratie zur Diktatur als auch die umgekehrte Variante, wiewohl in der Forschung der Übergang zur Diktatur häufig vernachlässigt wird. Im Gegensatz zu den Begriffen Regimewechsel (setzt keine Änderung der Herrschaftsform voraus) und Transformation (umfasst alle Veränderungen, also einschließlich der Dimension Systemwechsel) scheint »Systemwechsel « für die Beschreibung der Gesamtheit unterschiedlicher Transformationswege am besten geeignet. Vgl. zu Grundlagen und Grundbegriffen der Systemwechselforschung Marianne Kneuer, »Transformationsforschung, Systemwechselforschung, Demokratieforschung « in: Irene Gerlach / Eckhard Jesse / Marianne Kneuer / Nikolaus Werz (Hg.), Politikwissenschaft in Deutschland, Baden-Baden 2010, S. 265-290.

2 Vgl. statt vieler aktuell dazu Christoph Kleßmann, »Kommunismus im halben Land. Aufstieg, Charakter und Niedergang der DDR « in: Osteuropa 63 (2013) 5/6, S. 175-189, hier S. 188 f.

3 Hans Modrow, »Den Maueröffner Schabowski gibt es nicht « in: Eckhard Jesse / Thomas Schubert (Hg.): Zwischen Konfrontation und Konzession. Friedliche Revolution und deutsche Einheit in Sachsen, Berlin 2010, S. 189-207, hier S. 197 f. 
In fast allen früheren sozialistischen Blockstaaten ringen heute Vertreter aus Politik und Publizistik, aus Gesellschaft und Wissenschaft um die Deutungshoheit über die Systemwechselprozesse von 1989/90. Der wesentliche Grund für die unterschiedlichen Interpretationen der - je nach Perspektive - friedlichen, samtenen oder verhandelten Revolutionen ${ }^{4}$ liegt auf der Hand: dem weitgehenden Verzicht auf Gewalt und die schrittweise Aufgabe des Diktaturcharakters. Doch lässt sich die Rolle der SED-Spitze allein mit dem friedlichen Charakter des Systemwechsels und seiner Folgen, der erfolgreichen Übertragung der bundesdeutschen Demokratie auf das Gebiet der ehemaligen DDR, erklären und als solche positiv (um-)deuten? Einfach gefragt: Die Regimeelite vermied die »chinesische Lösung «, aber befürwortete sie zugleich Freiheit und Demokratie?

Um die Frage nach dem (Nicht-)Beitrag der Parteielite an der Demokratisierung im östlichen Deutschland beantworten zu können, gilt es zunächst, Unterscheidungen innerhalb der »alten Eilte« vorzunehmen (2.). Für eine solche Typologisierung werden die jeweiligen SED-PDS-Parteispitzen auf ihre zentralen Ziele und Strategien hin untersucht - entlang der in der Systemwechselforschung geläufigen Unterteilung in drei Transformationsphasen (Ende des alte Regime, Institutionalisierung und Etablierung bzw. Konsolidierung der Demokratie). ${ }^{5}$

Die Vorteile eines solchen Vor- und Abspanns gegenüber der Fixierung auf den Jahren 1989/90 sind offensichtlich: Zum einen sind nicht nur die Positionen und Strategien in der »heißen « Phase von Relevanz, sondern ebenso, ob sich bereits vor dem Machtverlust der SED erste Anzeichen einer politischen Öffnung feststellen lassen und inwieweit die Etablierung der Demokratie nach dem Abschluss der Institutionalisierung von der Erben der früheren Elite unterstützt wurde oder nicht. Zum anderen lassen sich entlang der drei Abschnitte Kontinuität und Wandel der Parteieliten analysieren. Dafür bietet sich eine chronologische Untersuchung entlang der drei Transformationsphasen mit zum Teil wiederkehrenden, zum Teil nur in bestimmten Zeiträumen relevanten Analysedimensionen an. Diese konzentriert sich auf drei wesentliche Bereiche: 1. auf die interne Entwicklung der Parteieliten, 2. auf das Verhältnis zu den eigenen Funktionseliten und den oppositionellen Gegeneliten und 3. auf spezifische Faktoren der drei Transformationsphasen (politische Schlüsselereignisse, Institutionenbildung bzw. demokratische Wahlen und Vergangenheitsbewältigung).

4 Siehe zu den Revolutionsbegriffen im Zusammenhang mit den Systemwechseln im (post)sozialistischen Osteuropa Stefan Auer, »Das Erbe von 1989. Revolutionen für Europa « in: Osteuropa 54 (2004) 5/6, S. 31-46.

5 Siehe zur Phaseneinteilung von Systemwechselprozessen Wolfgang Merkel, Systemtransformation. Eine Einfübrung in die Theorie und Empirie der Transformationsforschung, 2. Aufl., Wiesbaden 2010, S. 93-127. 


\section{Systemwechsel und die Rolle der Eliten}

Obwohl verschiedene Theorieansätze zur Erklärung von Systemwechselprozessen existieren, ${ }^{6}$ gelten die Eliten als die Schlüsselakteure der Demokratisierung: Ihre Interessen, Präferenzen, (Fehl-)Perzeptionen, Strategien und Kosten-Nutzen-Kalküle entscheiden über den Erfolg oder Misserfolg der Demokratisierung. ${ }^{7}$ Jedoch scheint es wenig sinnvoll, in Abgrenzung zu den neuen demokratischen Kräften für den gesamten Zeitraum des Systemwechsels von der »alten Elite «bzw. der »Regime- oder Machtelite $z$ zu sprechen, da sie dies nach dem Ende ihres Machtmonopols meist nicht mehr ist. Dem trägt der Terminus der »Parteielite « Rechnung. Er lässt sich für die gesamte Zeitspanne - vom Anfang des Endes der autokratischen Systeme bis zur Etablierung der Demokratien anwenden, und er umfasst beide Perspektiven: die Parteizirkel, die unter den Bedingungen des Spätsozialismus zugleich exklusive Machtelite waren, ebenso wie jene Kräfte, die nach dem Ende der Regime die Führung der einstigen Einheitsparteien behielten oder übernahmen, gesamtgesellschaftlich aber allenfalls einen Teil der politischen Elite repräsentierten.

In der Systemwechselforschung etabliert ist die Differenzierung der Eliten in »Hardliner « und Softliner ${ }^{8}$ Erstgenannte lehnen die Öffnung des politischen Systems ab. Dabei setzen sie auf risikointensive Strategien, auf verschärfte Repressionen, oder umgekehrt auf die Errichtung einer pseudodemokratischen Fassade, die aber nicht den hierarchischen und diktatorischen Charakter des Systems in Frage stellt. Sie halten reformunwillig am autokratischen Regime fest, sei es aus Opportunismus, um ihre Positionen und Privilegien zu sichern, sei es aus tatsächlicher Überzeugung, weil sie die Demokratie als Störfaktor innerhalb ihres politischen Weltbildes empfinden. Auch nach der Etablierung demokratischer Verfahren findet sich der Kern dieser Gruppe nicht mit den politischen Veränderungen ab, sondern versucht - falls er über die entsprechenden Machtmittel verfügt -, durch einen Putsch oder eine Verschwörung den Systemwechsel rückgängig zu machen.

Soft- und Hardliner lassen sich nicht immer eindeutig unterscheiden. Was die »Reformer « von den »Orthodoxen « unterscheidet, ist ihr wachsendes Bewusstsein, dass sie nur durch die Öffnung des politischen Systems ihren gesellschaftlichen Einfluss bewahren können. Sie stehen für risikovermeidende Strategien, fordern mehr politische Frei-

6 Vier theoretische Grundlinien der Transformationsforschung lassen sich unterscheiden: 1. Systemtheorien, die Transformationsprozesse mit den Veränderungen in Gesellschaft und Ökonomie erklären; 2. Strukturtheorien, welche den Aufbau des Staates und die Frage sozialer Klassen betonen; 3. Kulturtheorien, die auf die religiösen und mentalen Prägungen der Gesellschaften abzielen; 4. Akteurstheorien, die auf das konkrete politische Handeln der Protagonisten eines Systemwechsels verweisen. Vgl. als besten Überblick ebd., S. 67-88.

7 Vgl. ausführlich Ellen Bos, »Die Rolle von Eliten und kollektiven Akteuren in Transitionsprozessen« in: Wolfgang Merkel (Hg.), Systemwechsel 1. Theorien, Ansätze und Konzeptionen, Opladen 1994, S. 81-109.

8 Vgl. das Standardwerk der vergleichenden Systemwechselforschung von Guillermo O’Donnell / Philippe C. Schmitter / Laurence Whitehead (Hg.), Transitions from Autborian Rule, 4 Bde., Baltimore 1986, hier Bd. 3, S. 15-17. 
heiten und die Erhöhung der demokratischen Legitimation des Regimes zur Bewahrung der eigenen Macht. Zudem setzt sich innerhalb der »Softlinerfraktion« die Einsicht durch, dass sie im neuen System nur eine Chance haben, wenn sie die Öffnung mitgestalten und wenn die Einführung politischer Freiheiten nicht zu spät begonnen wird. Uneinig sind sich die Befürworter einer politischen Öffnung allerdings bei der Frage nach dem Umfang der Liberalisierung. Während ein Teil der Reformer einen begrenzten Pluralismus für eine bestimmte Zeit in Kauf zu nehmen bereit ist, wird bei anderen der dauerhafte Übergang zu einer demokratischen Ordnung angestrebt.

Vor dem Hintergrund sich schnell und häufig ändernder Akteurskonstellationen und -ziele in den verschiedenen Transformationsphasen überrascht, dass von der Systemwechselforschung bislang keine Versuche ausgingen, die grobe Differenzierung der alten Eliten zu verfeinern. Dabei weisen zumindest Teile der beiden Gruppen eine Reihe Gemeinsamkeiten auf, die einer trennscharfen Unterscheidung in »Hard « und »Soft« gegenübersteht. Nicht jeder Befürworter des alten Systems muss gegen eine anfängliche Öffnung sein, um die eigene Legitimität und Machtbasis zu stärken. Umgekehrt entpuppt sich nicht jeder Softliner nach dem Beginn der Öffnung als Verfechter der Demokratie. Vielmehr kann die Implementierung demokratischer Verfahren auf strategisch bzw. zweckrationales Verhalten zurückgehen, um sich bei der Etablierung des neuen Systems eine gute Ausgangssituation zu verschaffen. ${ }^{9}$ Sowohl die Hard- als auch die Softlinergruppe besteht folglich aus mindestens zwei Lagern, die mit ihren Pendants auf der jeweils anderen Seite eine Reihe von Ähnlichkeiten aufweisen.

Um eine aussagekräftige Unterscheidung vornehmen zu können, die differenziertere Formen von Parteieliten abdeckt und zugleich eindeutige Zuordnungen zu den Kategorien zulässt, scheint eine zweidimensionale Typologie besser geeignet zu sein als nur die Unterscheidung entlang der »Hard-Soft-Dichotomie«. Hierfür bietet sich eine Differenzierung der Eliten nach einerseits ihren Zielen und andererseits ihren Strategien an. Für die Frage nach den Zielen sind die ideologisch-programmatischen Systemvorstellungen entscheidend; strebt die Elite eine demokratische oder eine autokratische Ordnung an? Dem gegenüber soll ihre strategische Ausrichtung stehen: Erfolgt der Systemwechselprozess als exklusiver oder inklusiver Prozess, als Diktat oder Pakt. ${ }^{10}$ Daraus ergeben sich vier Typen von Parteieliten: 1. »harte« Antidemokraten (Orthodoxe), 2. »weiche « Antidemokraten (Pseudodemokraten), 3. »angepasste « Demokraten (Opportunisten) und 4. »überzeugte« Demokraten (Abbildung 1).

9 Siehe für die postsozialistischen Staaten ausführlich die Analyse von Philipp Harfst, Wablsystemwandel in Mittelosteuropa. Strategisches Design einer politischen Institution, Wiesbaden 2007.

10 Vgl. Terry L. Karl / Phillippe C. Schmitter, »Modes of Transition in Latin America, Southern and Eastern Europe« in: International Social Science Journal Nr. 128/1991, S. 269-284.

ZfP 60. Jg. 3/2013 
Abbildung 1: Parteieliten in Systemwechselprozessen nach Zielen und Strategien

\begin{tabular}{|l|c|c|}
\hline Ziel & Extrategie & Integration \\
\hline $\begin{array}{l}\text { Altes autokratisches } \\
\text { System }\end{array}$ & $\begin{array}{c}\text { »harte« Antidemokraten } \\
\text { (Orthodoxe) (1) }\end{array}$ & $\begin{array}{c}\text { »weiche« Antidemokraten } \\
\text { (Pseudodemokraten) (2) }\end{array}$ \\
\hline $\begin{array}{l}\text { Neues demokratisches } \\
\text { System }\end{array}$ & $\begin{array}{c}\text { »angepasste« Demokraten } \\
\text { (Opportunisten) (3) }\end{array}$ & $\begin{array}{c}\text { »überzeugte» } \\
\text { Demokraten (4) }\end{array}$ \\
\hline
\end{tabular}

Die orthodoxen Kräfte wollen das autokratische System um jeden Preis erhalten. Um den Zusammenbruch ihrer Herrschaft zu verhindern, setzen sie auf die Rücknahme möglicher Liberalisierungsmaßnahmen, die Mobilisierung ihrer eigenen Anhänger und den Ausschluss der Gegeneliten sowie auf den Einsatz des Repressionsapparates, gegenüber der Opposition und den Massen wie im eigenen Herrschaftsapparat. Sie sind von der Effizienz und (historischen) Richtigkeit des autokratischen Systems überzeugt, weswegen sie Reformen und Verhandlungen ablehnen. Setzt sich die Gruppe der »harten « Antidemokraten innerhalb der Parteielite durch und die Massenmobilisierung ihrer Gegner hält an, kann der Systemwechsel entweder nur in einem revolutionären Prozess erfolgen, oder es kommt zur Re-Stabilisierung der autokratischen Ordnung.

Auch die Gruppe der »weichen « Antidemokraten innerhalb der alten Elite steht für die Bewahrung des Regimes. Im Gegensatz zu den »harten «Orthodoxen versuchen diese jedoch nicht ausschließlich mit den Mitteln der Repression die Systemkrise zu überwinden, sondern sie reagieren pragmatisch und unideologisch auf den drohenden Machtverlust. Sie verweigern die Machtübertragung, sehen zugleich allerdings keine Erfolgsaussichten in der gewaltsamen Unterdrückung der Demokratiebewegung und setzen stattdessen auf den Ausbau politischer und sozioökonomischer Integrationsmechanismen. Dazu zählen kleine politische Freiheiten, z. B. die Zulassung einiger Oppositionsvertreter bei Wahlen (ohne dadurch die Mehrheitsverhältnisse in Frage zu stellen), eine begrenzte Presse- und Meinungsfreiheit oder auch massive wohlfahrtsstaatliche Maßnahmen (Wohnungsbau, soziale Sicherungssysteme, subventionierte Lebensmittel), um die Unterstützung der Gesellschaft wiederherzustellen. Sie hoffen, am Status quo einer Autokratie mit gewissen individuellen (weniger politischen) Freiheiten festhalten zu können. Gelingt es ihnen, Oppositionsbewegung und Bevölkerung derart zu instrumentalisieren und so das Ende der Massenmobilisierung zu erreichen, bleibt es bei einem autokratischen Regime oder es kommt zu einem graduellen Systemwandel - einer pseudodemokratischen Diktatur, nicht zur Demokratisierung.

Auf der anderen Seite der Parteieliten stehen die Befürworter eines System-, nicht unbedingt eines Machtwechsels, denn auch dieses Lager ist gespalten. Nicht allen »Softlinern« geht es um die Öffnung des politischen Systems. Vielmehr hat sich unter den »Opportunisten « die Einsicht durchgesetzt, dass sich die eigene Machtbasis nur bewahren lässt, wenn sie die Transformation selbst steuern und dabei wenig Beteiligung der 
Gegeneliten erlauben. Sie präferieren eine Dritte-Weg-Konzeption ${ }^{11}$ aus Elementen der alten und neuen Ordnung. Im Gegensatz zur Gruppe der »weichen« Antidemokraten, welche den Erhalt der alten Ordnung unter der Beteiligung einiger neuer Akteure anstreben, versuchen die »angepassten « Demokraten ein demokratisches System bei weitgehender Sicherung ihrer Machtbasis zu etablieren. Dafür setzen sie eher auf die Methoden der versteckten Manipulation als der offenen Repression.

Von den Parteieliten als Vorreitern der Demokratisierung lässt sich nur dann sprechen, wenn sie die angestrebte demokratische Staatsform wie die Integration der Bevölkerung und der Gegenelite vorbehaltlos unterstützten. Sie unterscheiden sich von der Gruppe der »angepassten « Demokraten, indem sie nicht nur versuchen, ihre Macht durch größere (demokratische) Legitimation zu erhalten, sondern unmittelbar zum Machtverzicht bereit sind. Sie öffnen das autokratische System nicht »fünf vor zwölf«, sondern selbstbestimmt. Sie befürworten die fundamentale Abkehr vom Ancien Régime, sie unterstützen den Dialog mit verschiedenen gesellschaftspolitischen Interessensgruppen und sie gehen durch die direkte Konfrontation mit den orthodoxen Kräften ein hohes persönliches Risiko ein. Setzen sich die »Demokratisierer « durch, vollzieht sich die Institutionalisierung der Demokratie unter der gleichberechtigten Beteiligung alter und neuer Kräfte. Sie erkennen das demokratische System vollständig an.

\section{Phasen der Systemwechsel von der Diktatur zur Demokratie}

\subsection{Das Ende der SED-Herrschaft}

Interne Veränderungen: Intern spricht viel für Kontinuität, wenig dagegen für einen Wandel der SED-Elite. Mit der Entmachtung Honeckers und seiner engsten Vertrauten war keine umfassende personelle Erneuerung an der SED-Spitze verbunden. Die »Putschisten im Politbüro «12 um Egon Krenz, Günter Schabowski und Willy Stoph gehörten teilweise jahrzehntelang dem engsten Führungszirkel an. Aus der Gruppe der Nachrücker um Modrow konnte ebenfalls keiner als »Alternative« gelten. Sie alle blickten auf erfolgreiche SED-Karrieren zurück, ${ }^{13}$ und keiner von ihnen hatte sich je durch eine selbst vorsichtig - kritische Haltung gegenüber der eigenen Machtpraxis hervorgetan. Eine selbstgesteuerte Öffnung der SED-Herrschaft gegenüber den reformorientierten Kräften aus der eigenen zweiten oder dritten Reihe - geschweige denn aus dem Lager der Opposition - blieb bis zum Fall der Mauer aus.

Nicht anders sieht es mit Blick auf die ideologische Erneuerungsfähigkeit in der SEDElite aus. Am Prinzip des demokratischen Zentralismus wurde ebenso wenig gerührt wie an der Ämterkumulation im Politbüro. Bis Anfang Dezember ließ die Führung keine

11 Siehe als Überblick zu dem facettenreichen Phänomen u. a. Alexander Gallus / Eckhard Jesse, "Was sind dritte Wege? Eine vergleichende Bestandsaufnahme in: Aus Politik und Zeitgeschichte Nr. 16-17/2001, S. 6-15.

12 So Günter Schabowski, Wir haben fast alles falsch gemacht. Die letzten Tage der DDR, Berlin 2009, S. 22.

13 Siehe im Einzelnen Gerd Meyer, Die DDR-Machtelite in der Ära Honecker, Tübingen 1991.

ZfP 60. Jg. 3/2013 
Zweifel an ihrer uneingeschränkten Führungsrolle in Staat und Gesellschaft, verlangte strenge Parteidisziplin von ihren Mitgliedern und verbot alle Formen innerparteilicher „Fraktionierung“. Sämtliche Personalentscheidungen und die Verteilung der höchsten Partei- und Staatsämter regelte die Spitze bis zu ihrem Untergang selbst. Einher ging dies mit einer weitgehenden Konstanz der ideologischen Grundlagen und programmatischen Eckpunkte der SED-Politik, wobei die Entmachtung Erich Honeckers erstmals eine Diskrepanz zwischen Zielen und Strategie des Politbüros offenbarte. Fehler der über Jahrzehnte als unfehlbar geltenden Staatspartei wurden erstmals (halbherzig) eingestanden und der Honecker-Clique angelastet mit dem Ziel, durch die »Wende « die »politische und ideologische Offensive wiederzuerlangen $\ll .{ }^{14}$ Gleichwohl hielt die Parteielite unbeirrt am uneingeschränkten Machtanspruch der SED fest.

Verbältnis zu Funktions- und Gegenelite: Für das Haltung zum Partei- und Sicherheitsapparat sowie zur Oppositionsbewegung lassen sich nur wenig Anhaltspunkte einer selbstbestimmten Systemöffnung erkennen. Die erfolgreiche Kaderpolitik der SED verhinderte bis zum Ende des Regimes, dass die gesellschaftlichen und ökonomischen Funktionseliten der Ineffizienz des Systems hätten entgegentreten können. Auch die Rollenverteilung zwischen SED und MfS blieb bis zum Zusammenbruch des SED-Regimes intakt. Dem wachsenden sozialen Druck begegnete das Politbüro vorrübergehend mit einer Zunahme an Repressionen. ${ }^{15}$ Das akribisch betriebene Berichtswesen des MfS wurde auf SED-Anweisung intensiviert, jedes Anzeichen »ideologischer Abweichung « der Parteiführung bzw. regionalen Leitungsgremien gemeldet und Demonstrationen so lang wie möglich verhindert. Um den 40. Jahrestag der DDR herum kam es vielerorts zum Einsatz des Repressionsapparates, und auch nach dem Sturz Honeckers änderte sich zunächst nichts grundsätzlich am Verhältnis von SED und MfS. Allerdings ergriff am 9. Oktober - dem Schlüsseldatum der friedlichen Revolution - kein Mitglied des Politbüros die Initiative, die bis dahin größte Montagsdemonstration in Leipzig gewaltsam aufzulösen. Dies hängt allerdings stärker mit dem Machtvakuum in den entscheidenden Stunden und weniger mit einem Nachgeben der SED zusammen. ${ }^{16}$

Bis zum Ende der ersten Systemwechselphase blieb die Oppositionsbewegung praktisch ohne jeden Handlungsspielraum. Im Verlauf der Systemkrise änderte die Parteielite allerdings ihre Strategie, ohne ihr Ziel, den Machterhalt, aufzugeben. Auf Repressalien und Unterwanderung folgte zunächst eine Verschärfung der Maßnahmen (Strafverfolgung). Nach Honeckers Sturz setzte die neue alte Führung um Krenz zunächst auf eine Vereinnahmung der Opposition durch »asymmetrische« Dialogangebote. ${ }^{17}$ Als sich die

14 Egon Krenz, Antrittsrede als Generalsekretär des ZK der SED am 18. Oktober 1989 in Berlin, unter http://www.cvce.eu.

15 Vgl. hier und im Folgenden statt vieler Andreas Malycha / Peter Jochen Winters, Geschichte der SED. Von der Gründung bis zur Linkspartei, München 2009, S. 302-360.

16 Vgl. ausführlich Michael Richter, Die Friedliche Revolution. Aufbruch zur Demokratie in Sachsen 1989/90, 2 Bde., Göttingen 2009.

17 Eine Ausnahme stellt das Dialogangebot von Hans Modrow, dem 1. Sekretär der SED-Bezirksleitung Dresden, an die »Gruppe der 20 « dar. Zu diesem Zeitpunkt gehörte er allerdings - trotz starken Rückhalts in der Partei, der Bevölkerung und nicht zuletzt in Moskau - noch nicht dem Politbüro als Machtzentrum des SED-Regimes an. 
Lage dadurch nicht beruhigen ließ, blieb ihr nur der Versuch, die Gruppen innerhalb der Opposition durch Anerkennung bzw. Nichtanerkennung gegeneinander auszuspielen. Mit einem »echten « Dialogangebot an den von der Führung akzeptierten Teil der Bürgerrechtsbewegung ging dies allerdings nicht einher.

Politische Schlüsselereignisse: Dass die Rede von der »chinesischen Lösung «mehr als eine Chiffre zur Verunsicherung und Schwächung der DDR-Protestbewegung war, belegen die Reaktionen der Parteiführung auf die gewaltsame Niederschlagung der Studentenproteste auf dem Tiananmen-Platz in Peking. ${ }^{18}$ Der Schulterschluss mit der chinesischen Führung sollte der wachsenden Opposition im eigenen Land signalisieren, auch die Machtelite der DDR würde im Fall der Fälle mit militärischen Mitteln reagieren. Aus dem Politbüro kamen ausschließlich Loyalitätsbekundungen gegenüber der KP Chinas (auch von den $»$ Maueröffnern ${ }^{19}$ Krenz und Schabowski), und selbst nach Honeckers Sturz und dem Fall der Mauer blieb bis zum Abgang der gesamten alten Elite jede Distanzierung von dem Massaker aus. Des Weiteren zeigte die Parteiführung später ihre Unfähig- bzw. Unwilligkeit zu einem Kurswechsel und zur Selbstkritik beim Krisenmanagement der Fluchtwelle über Ungarn und die bundesdeutschen Vertretungen in Prag und Warschau. Der Öffnung der ungarisch-österreichischen Grenze und den tausenden »Ausreisern « auf den Botschaftsterritorien begegnete die SED-Spitze mit verschiedenartigen Mitteln des Unterdrückungsapparates: durch die Verschärfung der Reiseregelungen, durch die Ausübung von Druck auf Angehörige und Bekannte, durch die Intensivierung der Grenzkontrollen und schließlich mit der Entscheidung zur vorübergehenden Schließung der DDR-Landesgrenzen. ${ }^{20}$ Ziel und Strategie deckten sich - es ging um die Bewahrung eines repressiven Systems mit den flexiblen Mitteln eines repressiven Systems.

Nur beim Fall der Mauer hatte sich das Kalkül der Parteielite gewandelt. ${ }^{21}$ Es war der letzte und hoffnungslose Versuch, das alte System durch die Gewährung der Reisefreiheit, aber ohne Machtverzicht zu bewahren. Auch wenn die Hoffnungen auf eine ReStabilisierung der SED-Macht unverständlich erscheinen, sind Intention und Auswirkung nicht zu verwechseln. Dass es der in Auflösung befindlichen Partei wenige Monate später nicht einmal mehr um die Frage der Macht, sondern »nur « noch um die Rettung einiger - vermeintlicher - DDR-Errungenschaften im Prozess der Deutschen Einheit gehen sollte, relativiert nicht den bis zum Systemzusammenbruch durchweg autokratischen Charakter der SED-Führung.

$18 \mathrm{Vgl}$. Anja Feege, Internationale Reaktionen auf den 4. Juni in der VR China. Solidarisierung, Schweigen und Sanktionen, Hamburg 1992, S. 66-69.

19 Vgl. etwa Peter Jochen Winters, »Maueröffner aus Versehen. Günther Schabowski wurde 80 Jahre alt « in: Deutschland Archiv 42 (2009), S. 11-13.

20 Siehe im Einzelnen Andreas Oplatka, Der erste Riss in der Mauer. September 1989 - Ungarn öfnet die Grenze, Wien 2009.

21 Vgl. ausführlich Hans-Hermann Hertle, Der Fall der Mauer. Die unbeabsichtigte Selbstauflösung des SED-Staates, Wiesbaden 1996.

ZfP 60. Jg. 3/2013 


\subsection{Vom Mauerfall bis zu den Volkskammerwablen - von der SED zur PDS}

Interne Veränderungen: Obwohl die Institutionalisierung der Demokratie von November 1989 bis zum März 1990 den deutlich kürzesten Zeitraum der drei Systemwechselphasen umfasst, sind für diesen Abschnitt die meisten Veränderungen an der Parteispitze anzuzeigen. Auf der personellen Ebene führte die Entwicklung bereits Anfang Dezember zum Bruch mit der einstigen DDR-Elite. Allein der Übergangskandidat Modrow saß im Politbüro (wenngleich nur wenige Wochen) wie im neuen Präsidium der Partei. Der Großteil der alten Garde wurde bis Ende Januar aus der SED-PDS ausgeschlossen; lediglich fünf ehemalige Politbüromitglieder verblieben in der Partei. Das neue Parteipräsidium bildeten vor allem einstige SED-Kader der mittleren Ebene. Diese setzten sich nach einer Reihe von Personalverschiebungen durch Aus- und Rücktritte sowie Ausschlüsse gegenüber eher demokratisch-sozialistisch (z. B. Wolfgang Berghofer) orientierten oder kommunistisch orientierten Flügeln durch. ${ }^{22}$ Wegen der Heterogenität an der Parteispitze sind die Zuordnungen nicht immer klar. Jene Kräfte, die ein autokratisches System befürworteten, stellten ebenso eine Minderheit dar, wie jene, die ein wertgebundenes Verhältnis zur Demokratie entwickelten. Dennoch blieben letztgenannte Positionen (auch) an der Parteispitze vorhanden, z. B. in Person Dietmar Kellers und Marlies Deneckes. Diese gilt es zu berücksichtigen. So lässt sich in Übereinstimmung mit dem Selbstverständnis die Mehrheit der Parteiführung um Gregor Gysi als »angepasste« Demokraten (Opportunisten) klassifizieren, wiewohl mit Ausschlägen in beide Richtungen.

Mit der strukturellen Wandlung von einer zentralistischen Staatspartei zu einer pluralistisch-demokratischen Partei tat sich die SED zunächst schwer, wie schon die anfangs beabsichtigte Beibehaltung des alten Namens belegt. Auch beim organisatorischen Umbau setzte die Partei aufgrund von Unentschlossenheit und Verunsicherung eher auf Wandel, statt eine umfassende Neuordnung anzustreben. Dies hatte vor allem materielle Gründe. Es galt, das Parteivermögen und Tausende von Arbeitsplätzen im Parteiapparat zu sichern. Die mittlerweile als SED-PDS, ab Februar als PDS bezeichnete Partei kam so mit einem Geburtsfehler zur Welt, der bis heute ein Dilemma für die innere Verfasstheit der SED-Nachfolgeparteien darstellt - auf der einen Seite als neue, demokratischlinke Kraft den radikalen Bruch mit dem SED-Regime demonstrieren zu wollen, auf der anderen Seite den Neuanfang auf eben diesen alten Strukturen gegründet zu haben. Trotz der selbstauferlegten Hypothek und einer deutlichen Machtfülle des Präsidiums gelang der Partei bis zur Volkskammerwahl die Transformation von einer monistischen Kaderpartei hin zu einer demokratisch-pluralistischen Mitgliederpartei. Ziel und Strategie der Führung waren identisch bei dem Versuch, möglichst viele verschiedene Strömungen einzubinden. Zugleich hatte sich eine offene und lebhafte Diskussionskultur entwickelt.

22 So Heinrich Bortfeldt, Von der SED zur PDS. Wandlung zur Demokratie, Bonn 1992; Gero Neugebauer / Richard Stöss, Die PDS. Geschichte. Organisation. Wäbler. Konkurrenten, Opladen 1996; dieses Urteil teilt auch einer der Beteiligen, der Kulturminister im Kabinett Modrow und spätere PDS-Abgeordnete Dietmar Keller, In den Müblen der Ebene. Unzeitgemäße Erinnerungen, Berlin 2012, S. 212-215. 
Existierte allerdings zu Zeiten des SED-Regimes ein Verbot der innerparteilichen Fraktionierung, widersprach dieser $\gg$ bis dahin deutlichste Bruch mit der alten Partei ${ }^{23}$ gerade wegen der innerparteilichen Heterogenität teilweise demokratischen Prinzipien. Der Versuch der Parteiführung, möglichst viele Mitglieder der unterschiedlichen internen Richtungen unter dem Dach der PDS vereinen zu können, hatte zur Folge, dass auch antidemokratische Kreise in der Partei eine neue alte Heimat fanden.

Auf ideologisch-programmatischer Ebene brach die Parteielite aus strategischen Gründen dagegen nicht vollends mit dem SED-Staat. ${ }^{24}$ Vor allem bei Fragen zur SEDVergangenheit versuchte die neu gewählte Parteispitze, die verschiedenen Flügel zu integrieren und blieb deswegen bei ihren inhaltlichen Entscheidungen gespalten und unentschlossen. Wegen dieses »Binnenpluralismus « ${ }^{25}$ fehlte es an einer einheitlichen Programmatik bzw. kam es zu Widersprüchen. Statt den wissenschaftlichen Anspruch der sozialistischen Lehre aufzugeben, versuchte die Parteiführung, einen Gegensatz zwischen der sozialistischen Theorie und der Herrschaftspraxis zu konstruieren. Zudem war im gesamten Wahlprogramm nicht einmal von der früheren Einheitspartei, nicht von den Verbrechen und Verfehlungen der SED-Diktatur oder ihrer Aufarbeitung die Rede. Trotz der Lippenbekenntnisse der Partei zur parlamentarischen Demokratie, zu Rechtsstaatlichkeit und zur sozialen Marktwirtschaft blieben ihre Aussagen in der Sprache abstrakt und inhaltlich vage.

Verbältnis zu Funktions- und Gegenelite: Die Bindung der Parteiführung an den Repressions- und Sicherheitsapparat wandelte sich nur zögerlich, auch nach dem personellen Wechsel von Krenz/Stoph zu Gysi/Modrow. Einerseits galt es, die Erneuerung der SED-PDS gerade in dem sensiblen Bereich MfS unter Beweis zu stellen. Andererseits zählten die Angehörigen der Staatssicherheit zur Kernklientel der Partei, auf deren Unterstützung die Führung nicht ohne weiteres verzichten wollte. Sie entschied sich für einen Mittelweg. Statt Beibehaltung oder Abschaffung wurde die Staatssicherheit in »Amt für Nationale Sicherheit « (AfNS) umbenannt; dies ging jedoch nicht mit einem umfassenden personellen und funktionalen Wandel einher. ${ }^{26}$ Erst durch den Druck der Öffentlichkeit, nachdem erhebliche Teile heikler Akten vernichtet und das Staatssicherheits-Vermögen verschwunden waren, fügte sich die Parteielite in das Unvermeidliche. Sie stimmte Mitte Dezember der AfNS-Auflösung zu. Jedoch setzte die Parteielite nach dem Jahreswechsel weiter auf ihre Verzögerungsstrategie. Sie blieb die Interessenvertretung der früheren MfS-Mitarbeiter. Wegen des eigenen Überlebenskampfes gab es für die PDS-Führung jedoch - außer einige materielle Sicherheiten für einen Teil des Appa-

23 Eckhard Jesse/Jürgen P. Lang, Die Linke - der smarte Extremismus einer deutschen Partei, München 2008, S. 35 f.

24 Statt vieler die Beiträge, Reden und Dokumente der PDS in: Wablparteitag. Progressiv - produktiv-pro DDR (24./25. Februar 1990), Berlin 1990; Lothar Bisky / Jochen Czerny / Herbert Mayer / Michael Schumann (Hg.), Die PDS - Herkunft und Selbstverständnis, Berlin 1996.

25 So Sebastian Prinz, Die programmatische Entwicklung der PDS. Kontinuität und Wandel der Politik einer sozialistischen Partei, Wiesbaden 2010, S. 31.

26 Vgl. hier und im Folgenden die Neuauflage des Standardwerks zum MfS von Jens Gieseke, Die Stasi. 1945-1990, München 2011, S. 254-269.

ZfP 60. Jg. 3/2013 
rates - nicht viel zu retten. Die Bereitschaft zur Auflösung und -deckung der Staatssicherheitspraktiken stieg in dem Maße, wie der eigene Machtverlust näherrückte.

Ähnlich zögerlich verhielt sich die Parteiführung bei der Anerkennung und Beteiligung der Opposition. ${ }^{27}$ Bis Anfang Dezember versuchte sie, ein Dialogforum, wie den Runden Tisch in Polen, zu verhindern. Erst nach dem Rücktritt des gesamten Politbüros beugte sich die neue Spitze dem anhaltenden Druck der Öffentlichkeit. Gleichwohl war die »Salamitaktik « zur Machtbeteiligung der neuen politischen Konkurrenz in den ersten Wochen kein Ausdruck von innerer Überzeugung, sondern vielmehr dem Machtvakuum in einer Phase großer politischer Ungewissheit geschuldet. Die Hoffnungen der Parteielite richteten sich darauf, die Opposition durch Beteiligung in die Verantwortung einbinden zu können, so eine größere Legitimation für die eigenen Entscheidungen zu erhalten und damit den Machtverlust zu verhindern. Dafür wurde am 5. Februar die »Regierung der Nationalen Verantwortung « gebildet. Das Kalkül der Parteielite ging auf, die linke Opposition wurde wegen ihrer Instrumentalisierung durch die PDS geschwächt. Gleichwohl konnte die »Umarmung « des politischen Gegners den Machtverlust der Partei nicht verhindern.

Institutionenbildung: Auch die Bildung demokratischer Institutionen begann erst nach der Auflösung des Politbüros. Der Vorschlag der Krenz-Administration zur Umgestaltung der Verfassung war nicht mit einem Demokratisierungsangebot verbunden und zielte auf die Rettung des SED-Regimes, nicht auf die Öffnung der Gesellschaft. ${ }^{28}$ Wegen der fehlenden Legitimität der Volkskammer und durch die sich abzeichnende Entwicklung in Richtung deutsche Einheit kam es beim Erneuerungsversuch der DDRVerfassung zur Einbindung des Runden Tisches. Der Sonderfall »Wiedervereinigung « führte zu einem Strategiewechsel der Parteielite, die sich mit einem »radikaldemokratischen « Verfassungsentwurf als Hüterin der DDR inszenierte. Der Modrow-Regierung ging es dabei vor allem um die Abwehr der befürchteten westdeutschen Dominanz im Einigungsprozess, gleichwohl fehlte ihr der Wille zum Verfassungskonsens. Dennoch war es der gescheiterte Versuch um eine demokratisch und rechtsstaatlich einwandfrei zustande gekommene DDR-Verfassung.

Bei der Transformation der Volkskammer fällt die Bewertung differenziert aus. ${ }^{29}$ Vor allem die Ebene der Repräsentation innerhalb der Volkskammer widersprach bis zum Ende der Institutionalisierungsphase den Prinzipien von demokratischen Volksvertretungen. Bei der Gesetzgebungs- sowie der Wahl- und Kreationsfunktion entfaltete die

27 Siehe ausführlich Uwe Thaysen, Der Runde Tisch. Oder: Wo blieb das Volk? Der Weg der $D D R$ in die Demokratie, Opladen 1990; ders. (Hg.), Der Zentrale Runde Tisch der DDR. Wortprotokoll und Dokumente, 5 Bde., Wiesbaden 2000.

28 Vgl. ders./Hans Michael Kloth, »Der Runde Tisch und die Entmachtung der SED. Widerstände auf dem Weg zur freien Wahl «, in: Deutscher Bundestag (Hg.), Materialien der Enquete-Kommission "Aufarbeitung von Geschichte und Folgen der SED-Diktatur in Deutschland ", neun Bände in 18 Teilbänden, Band VII/2, Frankfurt a. M./Baden-Baden 1995, S. 1706-1826.

29 Vgl. Hans Michael Kloth, Vom "Zettelfalten" zum freien Wählen. Die Demokratisierung der DDR 1989/90 und die »Wablfrage«, Berlin 2000, S. 562 f.; Uwe Kranenpohl, »Als die Volkskammer (fast) zum Parlament wurde ... >Sozialistischer Parlamentarismus in der DDR 1989/90 « in: Zeitschrift für Parlamentsfragen 41 (2010), S. 121-141. 
Volkskammer nach der Streichung des Machtmonopols der SED rege Aktivitäten, die jedoch nur teilweise im Einklang mit den Absichten der Parteielite standen. Nicht zuletzt wegen ihres Kontrollverlustes in der Volkskammer versuchte die SED-PDS-Führung, wesentliche Entscheidungen in kleineren, weil besser kontrollierbaren Institutionen (Runder Tisch, Ministerrat) zu regeln. Das gelang ihr teilweise; umgekehrt setzte die Partei nicht auf die Verhinderung abweichender Positionen. Insbesondere in den Bereichen Kontrolle und Kommunikation entfaltete die Volkskammer nach dem Zusammenbruch des SED-Regimes rege Aktivitäten. Auch in diesen Bereichen wurde die Spitze nicht zuletzt aus den eigenen Reihen - für ihre Hinhaltestrategie kritisiert. So gelang es nicht, die Volkskammer in ein „echtes“ Parlament umzuwandeln, was einerseits an der fehlenden Legitimität des Plenums und der Konkurrenz durch den Runden Tisch, andererseits an der anfänglichen Gegenwehr und Verzögerung durch die SED-PDS-Spitze lag.

Freiwilligkeit der Parteispitze war auch auf dem Weg zu den Volkskammerwahlen im März 1990 noch über das Ende des SED-Regimes hinaus Fehlanzeige. Das Politbüro unter Krenz setzte auf die Verzögerung von Neuwahlen. Mit der personellen Erneuerung der SED-PDS wandelte sich die Strategie. Der neue Parteivorstand um Gysi und Berghofer trat für unverzügliche Neuwahlen ein. Ihm ging es nicht mehr um die Rettung der Macht, sondern um die Rettung der DDR. Der Demokratisierungswille der SED-PDS zeigte sich, indem sie die Ausarbeitung eines Wahlgesetzes durch die Volkskammer veranlasste, allerdings nicht ohne ihren organisatorischen Vorsprung strategisch auszunutzen. Vor allem bei den Fragen zum Wahltermin und zur Herstellung der Chancengleichheit setzte sie statt auf Konsens beharrlich auf Regelungen zum eigenen Vorteil. Das spricht gegen eine aktive Vorreiterrolle der SED-Elite beim Prozess der Institutionalisierung demokratischer Verfahren.

\subsection{Die PDS und die Demokratie der Bundesrepublik Deutschland}

Interne Veränderungen: Waren antidemokratische Einstellungen an der PDS-Spitze im Institutionalisierungsabschnitt der Demokratie auf dem Rückzug, ließ sich mit dem Machtverlust nach den Volkskammerwahlen im März eine »Verschlechterung « ihrer demokratischen Verfasstheit feststellen. Wegen der internen Heterogenität blieben zwar auch demokratische Akteure in der Parteiführung (z. B. Helga Adler, André Brie, Karin Dörre, Dietmar Keller) präsent, allerdings verschob sich der Kurs zunehmend in Richtung Systemablehnung. Im Gegensatz zur SED, deren Ende nach dem Wegfall der Unterstützung durch die Sowjetunion aus Überalterung ihrer Führung resultierte, fehlte es der PDS-Elite an personeller Kontinuität. Die hohe Fluktuation hatte vier wesentliche Ursachen: 1. geringe berufliche Chancen und politische Perspektiven, 2. neue Führungspersonen durch die Westausdehnung der Partei, 3. Unzufriedenheit (aus verschiedenen Gründen) mit der politischen Entwicklung der PDS, 4. Rücktritte wegen der Diskreditierung von PDS-Spitzenleuten durch ihre MfS-Verstrickungen. Für den Demokratisierungswillen der Parteielite spricht die Zurückdrängung von politisch diskreditierten 
Führungspersönlichkeiten; dagegen der Verbleib von zahlreichen Inoffiziellen Mitarbeitern des MfS, der Einflussverlust der demokratischen Strömungen (die frustriert die Partei verließen) und die Integration westdeutscher Führungskräfte aus dem Milieu der dogmatischen Linken und der K-Gruppen. Zugunsten der Einbindung möglichst vieler interner Strömungen verzichtete die Parteiführung auf eine klare Hinwendung zum demokratischen Verfassungsstaat der Bundesrepublik Deutschland.

Die Integration verschiedener Strömungen ging mit der strukturellen Umgestaltung der PDS einher. Die Parteiführung versuchte, den Verfall durch die Anbindung verschiedener Milieus zu verhindern, so neue Anhänger zu gewinnen, sich für Bündnisse inner- und außerhalb des Parteiensystems zu öffnen sowie ihre gesellschaftliche Verankerung voranzutreiben. Dies widersprach zum Teil den Demokratisierungsbemühungen der frühen PDS. Trotz Spannungen gelang es der PDS-Führung, die innerparteilichen Faktionen zusammenzuhalten. Meinungsverschiedenheiten und Widersprüche wurden nicht zugunsten einer einheitlichen Parteilinie zurückgestellt, sondern als Zeichen inneren Pluralismus interpretiert. So fanden nicht nur demokratische Zirkel, sondern auch klar antidemokratisch eingestellte Kräfte, wie die Kommunistische Plattform, die AG Junge GenossInnen und Cuba Sí, in der PDS eine politische Heimat. ${ }^{30}$ Der Demokratisierungsdrang der PDS-Führung ist auf organisatorischer Ebene folglich ambivalent zu bewerten. Einem hohen Maß an innerparteilicher Pluralität und Demokratie steht die Heterogenität der verschiedenen Bewegungen gegenüber, und damit die Unwillig- bzw. Unfähigkeit der Parteiführung, sich uneingeschränkt zur demokratischen Verfasstheit des politischen Systems zu bekennen.

Auf ideologisch-inhaltlicher Ebene verhinderte der strukturelle »Geburtsfehler« der Partei, der Klientelpflege den Vorrang gegenüber einem inhaltlichen Neuanfang gegeben zu haben, ein demokratisches Programm. Die Partei entschied sich-vor die Wahl gestellt, demokratische Positionen zu beziehen und einen Teil ihrer Anhängerschaft zu verprellen oder zugunsten einer breiten Integration einen Mittelweg aus demokratischen und extremistischen Einstellungen zu gehen - für die letztgenannte Option. Durch die Konzeption eines so bezeichneten Dritten Weges und die PDS-Rolle als Bewahrerin von tatsächlichen und vermeintlichen - DDR-Errungenschaften gelang es der Partei, die inneren Reihen zu schließen. Verklärung, Ostalgie und Antikapitalismus wurden zum einigenden Band der PDS-Spitze und zur Abgrenzung gegenüber dem demokratischen Modell der Bundesrepublik genutzt.

Verbältnis zu den neuen und alten Eliten: Die Beziehung der PDS-Führung gegenüber den Macht- und Funktionseliten des alten Regimes blieb ambivalent. Zwar nutzte die Partei die klare Schuldzuweisung gegenüber dem Politbüro, um sich selbst als erneuerte Kraft zu stilisieren. In dem Maße aber, wie die tatsächliche oder gefühlte soziale Deklassierung eines Großteils der PDS-Wählerschaft wuchs, nahm die Bereitschaft zu, als Verteidigerin der früheren Eliten in Erscheinung zu treten. Die juristische Aufarbeitung des

30 Vgl. Jesse / Lang, Die Linke, aaO. (FN 23), S. 33-45; Eckhard Jesse, »Extremismus in Deutschland « in: ders. / Tom Thieme (Hg.), Extremismus in den EU-Staaten, Wiesbaden 2011, S. 83-98, hier S. 92-94. 
DDR-Unrechts, gegen die Machthaber wie in den sogenannten Mauerschützenprozessen, wurde von der gesamten PDS-Führung abgelehnt, wenngleich aus unterschiedlichen Beweggründen. Ging es den reformorientierten Kräften stärker um die Pflege ihrer Klientel, galten den Hardlinern die politischen Strafprozesse als »Schauprozesse« und »politische Verfolgung «. 31

Im Parteiensystem wurde die PDS isoliert. Als Reaktion auf die Ablehnung aller Bundestagsparteien ging sie selbst zunehmend auf Distanz. Hieß es im Programm von 1990, man strebe gegenüber der SPD ein partnerschaftliches Verhältnis an und wolle eine konstruktive Oppositionsrolle spielen, so reagierte die PDS-Elite auf ihre Ausgrenzung, indem sie sich zunehmend abschottete. Vor allem gegenüber den „bürgerlichen“ Regierungsparteien verhärtete sich der Konfrontationskurs. Nur langsam entspannte sich das Verhältnis zu SPD und Bündnis 90/Grüne durch die zaghafte gegenseitige Annäherung auf Landesebene. Der Polarisierungsgrad im deutschen Parteiensystem nahm durch den Einzug der Sozialisten zu, die Segmentierung dagegen ab. ${ }^{32}$ Auch das Verhältnis der Partei zu den gesellschaftlichen und wirtschaftlichen Eliten des Landes schwankte zwischen fundamentaler Ablehnung und partieller Zusammenarbeit.

Vergangenheitsbewältigung: Die Auseinandersetzung mit der Vergangenheit wurde für die PDS-Spitze von Anfang der 1990er Jahre an zum Dauerthema. ${ }^{33}$ Die Folge der einseitigen Schuldzuweisungen in Richtung Politbüro war eine zunehmende Verklärung der Konzeption des Realsozialismus im Allgemeinen und der DDR-Geschichte im Besonderen. Die enge Verbindung von (N-)Ostalgie mit den politischen Biographien der PDS-Anhänger bestimmte die Strategie der Führung bei Fragen zur Vergangenheit. Differenzierungen zwischen »denen da oben « und einfachen »Parteisoldaten« wurden zum Erklärungs- und Rechtfertigungsmuster und zum Totschlagargument gegenüber »pauschaler Schwarz-Weiß-Malerei«. So präsentierte sich die PDS-Elite als Kraft der Erneuerung mit dem Ziel, die als positiv empfundenen Errungenschaften der DDR zu verteidigen. Den Vergleich der beiden deutschen Staaten benutzte die PDS-Spitze mit dem Verweis auf die - tatsächlichen oder angeblichen-DDR-Verdienste dazu, das alte System aufzuwerten. Die »antifaschistisch-demokratischen « Veränderungen der DDR und die Gestaltung einer sozialistischen Gesellschaft galten, trotz aller Verfehlungen, als nach wie vor berechtigter Gegenentwurf zum »antidemokratischen « Kapitalismus im Westen.

Die Frage nach der Aufarbeitung der SED-Diktatur führte im Gegensatz zur innerparteilichen Einigkeit bei der Bewertung der DDR zu heftigen Kontroversen. Die Mehrheit der PDS-Elite befürwortete die Interpretation der Schuldbefreiung: Nicht der So-

31 Vgl. ausführlich Tom Thieme, Systemwechsel in der DDR und in Ostmitteleuropa. Die Rolle der Parteieliten im Prozess der Demokratisierung im Vergleich (Habilitationsschrift), erscheint Wiesbaden 2014.

32 Vgl. Oskar Niedermayer, »Nach der Vereinigung. Der Trend zum fluiden Fünfparteiensystem « in: Oscar W. Gabriel / ders. / Richard Stöss (Hg.), Parteiendemokratie in Deutschland, 2. Aufl., Bonn 2001, S. 107-127.

33 Vgl. zur internen Auseinandersetzung mit der SED-Vergangenheit Dietmar Keller / Matthias Kirchner (Hg.), Zwischen den Stüblen. Pro und Kontra SED, Berlin 1993; Dietmar Keller u. a. (Hg.), Ansichten zur DDR-Geschichte, 11 Bde., Eggersdorf 1993-1998.

ZfP 60. Jg. 3/2013 
zialismus an sich und die Mitglieder der SED seien für die autokratische »Deformation « der DDR verantwortlich gewesen, sondern allein die zentralistisch-repressive Elite im Politbüro. Nur eine Minderheit beklagte die halbherzige Aufarbeitung der eigenen Parteigeschichte und wehrte sich gegen die Versuche vieler ehemaliger Funktionäre, die Parteihierarchie als Ausrede für das eigene Versagen heranzuziehen. Diese Gruppe konnte sich als innerparteiliche Minderheit allerdings nicht für ein eindeutiges Bekenntnis zur gemeinsamen Aufarbeitung mit allen demokratischen politischen Kräften durchsetzen. Ihre Vertreter wandten sich letztlich gegen die Arbeit, Aufgaben und Ergebnisse der Enquete-Kommission »Aufarbeitung « - mit ihrem Konfrontationskurs blieben einzig sie bei der Auseinandersetzung mit der Diktaturgeschichte »außen vor «.

Den Systemwechsel 1989/90 mit der deutschen Einheit bewertete das Machtzentrum der PDS weitgehend negativ. Auch hier griff die Parteiführung zu ihrem bewährten Mittel, die unterschiedlichen Dimensionen des Wandels differenziert zu beurteilen. Auf politischer Ebene wurde das - als deformiert geltende - Sozialismusexperiment als grundsätzlich legitim betrachtet: Das 40-jährige Regiment der Partei- und Staatsführung habe es zum Scheitern gebracht. Die wirtschaftlichen und sozialen Dimensionen des Systemwechsels bewertete die PDS-Spitze dagegen weitgehend negativ. Vor allem die DDR und ihre »Errungenschaften « wurden mit wachsendem zeitlichen Abstand zum Systemwechsel zunehmend positiv gesehen; dagegen verblasste die kritische Distanz zum autokratischen System. Der Einschätzung Dietmar Kellers, »die PDS war im März 1990 schon deutlich weiter «, ${ }^{34}$ ist wenig hinzuzufügen - außer: Sie befand sich auch nie stärker in der Defensive als Anfang 1990.

\section{Fazit}

Die Gesamtbilanz der SED-PDS-Parteielite während der Demokratisierung in der DDR fällt ambivalent aus. Die alte Garde unter Honecker reagierte auf die in- und externen Zerfallserscheinungen ihrer Herrschaft mit stoischer Beharrung. Mit dem Verweis auf die bessere wirtschaftliche Lage in der DDR gegenüber den nach Reformen drängenden Polen wähnte sie sich auf dem richtigen Weg. Sie hielt an ihrer uneingeschränkten Führungsrolle in Staat und Gesellschaft fest, sie verlangte strenge Parteidisziplin von ihren Mitgliedern und festigte die ideologischen Grundlagen und programmatischen Eckpunkte ihrer Politik. Die Oppositionsbewegung blieb bis zum Sturz Honeckers ohne Handlungsspielraum. Erst danach entstand eine Inkongruenz von Zielen und Strategie innerhalb der SED-Spitze. Obwohl die »Wende«, die nicht mehr als die Lage beruhigen sollte, unter Krenz und Co. erstmals zu Zugeständnissen führte (Kritik an Honecker, asymmetrische Dialogangebote), zielte das Politbüro auf die Festigung seines uneingeschränkten Machtanspruchs, nicht auf die Öffnung des Regimes. Freie Wahlen verkündete die neue alte Führung am Tag des Mauerfalls - als die »Messe gelesen war «. Bis zum Verlust ihres Machtmonopols dominierten in der SED »harte « Antidemokraten, deren Beitrag am friedlichen Charakter des Systemwechsels keiner demokratischen Überzeu-

34 Ders. im Interview mit dem Verfasser am 26. April 2012 in Lichtenwalde bei Chemnitz. 
gung geschuldet war, sondern vor allem auf die Fehlkalkulation der Honecker-Nachfolger zurückging, das Blatt noch zu ihren Gunsten wenden zu können.

Mit der Umbenennung der SED in SED-PDS und schließlich PDS kam es zum (fast) vollständigen Austausch der einstigen DDR-Führung, doch verhinderte die Heterogenität der neuen Parteispitze den klaren Bruch mit dem alten System. Dies wirkte sich auf die organisatorische und programmatische Erneuerung aus. Beim Umbau setzte die Partei aufgrund von Unentschlossenheit und Verunsicherung eher auf Wandel, statt eine umfassende Neuordnung anzustreben. Ziel und Strategie waren identisch bei dem Versuch, möglichst viele verschiedene Strömungen einzubinden. Die neue Führung versuchte, sie unter dem Dach der PDS zu vereinen mit der Folge, dass auch offenkundig antidemokratische Kreise in der Partei eine neue alte Heimat fanden. Auch auf ideologisch-programmatischer Ebene kam es aus strategischen Gründen (zur Sicherung ihrer Klientel) nicht zu einem radikalen Bruch mit dem SED-Regime. Durch die Integration der verschiedenen Flügel blieb eine einheitliche programmatische Linie aus. Statt den wissenschaftlichen Anspruch der sozialistischen Lehre aufzugeben, kultivierte die Parteiführung den Gegensatz zwischen sozialistischer Theorie und realsozialistischer Herrschaftspraxis. Auch im Verhältnis zur Opposition und bei der Genese demokratischer Institutionen entstand trotz umfassender Veränderungen kein wertgebundenes Verhältnis zur Demokratie. Die PDS-Spitze unterstützte zwar nun die Demokratisierung der DDR, doch befand sie sich während der Übergangsphase massiv in der Defensive. Zudem überlagerte die sich abzeichnende deutsche Einheit den Systemwechsel: Die PDS-Elite akzeptierte eine demokratische DDR, nicht die deutsche Einheit. Eine Führungsrolle übernahm die Parteielite während der Institutionalisierung nicht. Sie verzögerte die Auflösung der alten Partei- und Staatssicherheitsstrukturen, die Anerkennung und Beteiligung der Opposition und den Weg zu freien Wahlen. Erst unter dem anhaltenden Druck beugte sie sich den Forderungen der Öffentlichkeit - ihre »Salami-Taktik « war darauf ausgerichtet, nicht mehr an Macht abzugeben als notwendig. Strategie und Ziel deckten sich hier nicht. Durch die Beteiligung der Opposition erhoffte sich die PDS-Elite, diese in die Verantwortung einbinden zu können, um eine größere Legitimation für Entscheidungen zu erhalten und damit den Machtverlust zu verhindern. Dafür wurde die »Regierung der Nationalen Verantwortung " gebildet. Zwar wurde die Opposition durch die »Umarmung « der PDS geschwächt, die Parteispitze konnte durch die Entwicklung in Richtung deutscher Einheit ihren Machtverlust aber nicht verhindern. Insgesamt vollzog die SED-PDS während der Institutionalisierung ihren wohl deutlichsten Wandel von »harten Antidemokraten« zu »angepassten « Demokraten. Sie unterstützte die Demokratisierung - allerdings weniger der Demokratie wegen als vielmehr um ihrer selbst willen.

Die demokratische "Nagelprobe « in der abschließenden Etablierungsphase bestand die PDS-Elite nicht. Durch ihren Machtverlust und ihre Einflusslosigkeit auf den Prozess der deutschen Einheit zog sie sich in eine Nische zurück. Erstens verzichtete die Parteispitze zugunsten der Integration möglichst vieler parteiinterner Strömungen auf ein klares Bekenntnis zur Demokratie. Zweitens verstärkte die Suche nach neue Zielgruppen die Öffnung der Partei für antidemokratische Anhänger und Milieus - dies untergrub 
die Demokratisierungsbemühungen eines Teils der PDS-Elite. Drittens verhinderte die interne Heterogenität das Entstehen einer demokratischen Programmatik. Viertens blieb das Verhältnis der PDS-Führung zu den alten Funktions- und neuen Gegeneliten gespalten - die juristische Aufarbeitung der DDR lehnte sie ab. Im Parteiensystem wurde sie isoliert. Fünftens fehlte ihr der Willen zu einer konsensuellen Aufarbeitung mit den demokratischen politischen Kräften, den Systemwechsel wie die deutsche Einheit bewertete sie insgesamt als negativ. Dominierten bis zum Ende der ersten Systemwechselphase in der SED-Führung »harte " Antidemokraten und in Phase zwei »angepasste « Demokraten, lavierte die PDS-Spitze nun entlang ihrer internen Strömungen zwischen Demokratie und Extremismus.

Das Hauptverdienst der alten Parteielite lag darin, in den entscheidenden Stunden im Herbst 1989 nicht die Nerven verloren zu haben, obwohl aus der Sicht mancher alter Kader sie diese gerade verloren hatten. Daraus einen positiven Beitrag oder gar eine Vorreiterrolle im Demokratisierungsprozess abzuleiten, übersieht die Diskrepanz zwischen Intention und Wirkung. Im Fortgang der Transformation blieb die PDS-Spitze äquidistant gegenüber dem alten und dem neuen System. Die deutsche Einheit lehnte sie ab. Daher sind Äußerungen des heutigen Vorsitzenden der Links-Fraktion Gysi, seine Partei sei die einzige, die für die innere Einheit (bezogen auf die sozialen Unterschiede zwischen Ost und West) kämpfe, nicht mehr als das Zünden von Nebelkerzen. Das Motto »Vorwärts und nicht vergessen ${ }^{35}$ scheint für die eigene Geschichte in den Jahren des Umbruchs längst nicht für jeden Vertreter der Parteielite zu gelten.

\section{Zusammenfassung}

Obwohl die Systemwechselforschung seit dem Zusammenbruch des Kommunismus einen Boom erlebt hat und die »Schlüsselrolle der Eliten « weithin anerkannt ist, dominieren nach wie vor zeithistorische Studien gegenüber den politikwissenschaftlich-akteurszentrierten Analysen. Der Schwerpunkt dieses Aufsatzes liegt auf der sozialistischen Parteielite der DDR und deren (Nicht-)Beitrag zur Demokratisierung - vor, während und nach der friedlichen Revolution 1989/90. Die Untersuchung der »Parteieliten« erfolgt entlang drei zentraler Dimensionen: 1. interner Veränderungen, 2. für das Verhältnis zu den eigenen Funktionseliten und den oppositionellen Gegeneliten und 3. auf spezifische Faktoren der drei Transformationsphasen. Im Ergebnis kann - trotz der deutlichen Unterschiede zwischen den einzelnen Transformationsphasen - der SED-PDS-Elite ein allenfalls bescheidener Beitrag zur Implementierung der repräsentativen Demokratie auf dem Gebiet der ehemaligen DDR zugeschrieben werden. Sie unterstützte die Demokratisierung - allerdings weniger der Demokratie wegen als vielmehr um ihrer selbst willen.

35 So die erste Zeile des "Solidaritätsliedes « von Hans Eisler (Melodie) und Bertolt Brecht (Text) und Titel der aktuellen Veröffentlichung zum Geschichtsbild Der Linken/PDS von Christian Lannert: »Vorwärts und nicht vergessen «? Die Vergangenheitspolitik der Partei Die Linke und ibrer Vorgängerin PDS, Göttingen 2012. 


\section{Summary}

Despite the research of regime change has manifold developed since the collapse of communism and the "key role of the elites « is widely acknowledged, there is still a overweight of historical studies compared with political science actor-centered analysis. The focus of this article is on the socialist party elite of the GDR and their (non-)contribution to the democratization - before, during and after the Revolution of 1989/90. The study of "party elites « occurs along three key dimensions: 1) internal changes, 2) for the relationship to their own function elites on the one hand and the counter elites and the opposition on the other hand, and 3) to specific factors of the three phases of transformation. As a result - despite of the significant differences between the three periods of regime change - the partyelite of SED-PDS did hardly participate during the implementation of representative democracy in the territory of the former GDR. They supported democracy but less because of the the democray and more because of themself.

Tom Thieme, The SED-PDS-Elite and the regime change in the GDR

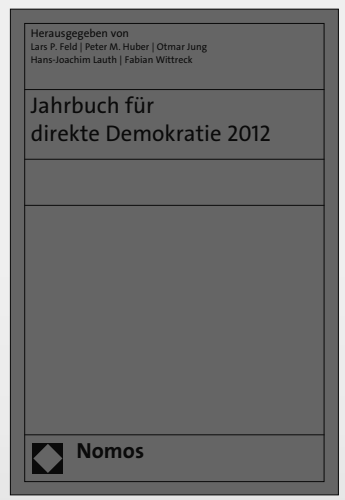

\section{Jahrbuch für direkte Demokratie 2012}

Herausgegeben von Lars P. Feld, Peter M. Huber, Otmar Jung, Hans-Joachim Lauth und Fabian Wittreck

2013, ca. 336 S., brosch., ca. 85,-€

ISBN 978-3-8487-0591-7

Erscheint ca. September 2013

Der nunmehr vierte Band des Jahrbuchs dokumentiert die aktuelle Entwicklung in Deutschland und den klassischen Referenzländern der direkten Demokratie (Schweiz und Vereinigte Staaten), nimmt aber auch die langjährige Praxis in Italien in den Blick. Das Jahrbuch enthält Rezensionen, eine nochmals erweiterte Bibliographie und dokumentiert die einschlägige Rechtsprechung.

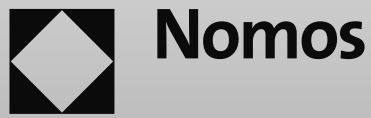

